\title{
Light- and Heavy-chain Deposition Disease (LHCDD): Difficulty in Diagnosis and Treatment
}

Key words: light- and heavy-chain deposition disease (LHCDD), PBSCT, renal failure

Light- and heavy-chain deposition disease (LHCDD), light-chain deposition disease (LCDD), and heavy-chain deposition disease (HCDD) are rare illnesses. A comprehensive review of the clinical features, immunopathology, and molecular biology of LCDD and related disorders was provided in 1992 by Buxbaum (1) and updated by Gallo et al (2). There is little information on the prognosis of patients with LHCDD treated with autologous stem cell transplantation (ASCT).

\section{Characteristics of LHCDD}

1) Unexplained proteinuria, renal failure, hematuria, or hypertension.

2) LCDD lacks the clinical characteristics of multiple myeloma, and occurs in younger ( $30-50$ years), predominantly female, patients.

3) A kind of monoclonal plasma cell disorder.

4) The finding of homogeneous serum and/or urinary immunoglobulin-related $\mathrm{M}$ protein by the agarose gel or immunofixation electrophoresis methods. LCDD-associated monoclonal Igs are predominantly kappa-type, whereas there is a predominance of lambda-type in AL amyloidosis.

5) In order to diagnose LCDD definitively, a kidney biopsy is essential. Nodular glomerulosclerosis resulting from the co-deposition of light-chains with extracellular matrix proteins is the pathologic hallmark. Skin biopsies revealed the pathologic deposits at the dermal-epidermal junction.

6) Activation of platelet-derived growth factor $\beta$ (PDGF- $\beta$ ) and transforming growth factor $\beta$ (TGF- $\beta$ ) may play a role in the mediating glomerurosclerosis and hyperplastic vasculopathy.

7) Major therapeutic efforts have been directed towards reducing the synthesis of $\mathrm{M}$ proteins. This can best be achieved by chemotherapeutic regimens that are effective in myeloma, such as melphalan-prednisone (MP), vincristine-doxorubicin-dexamethasone (VAD), and high-dose melphalan $\left(140-200 \mathrm{mg} / \mathrm{m}^{2}\right)$ in conjunction with autologous/allogeneic hematopoietic stem cell transplantation.

8) Renal transplantation in hemodialysis patients, who showed disease stability for more than one year and no evidence of clinically significant extrarenal deposition.

9) The overall prognosis remains poor, and death eventually results from infection and failure of vital organs (by the deposition in liver, heart, and lungs) targeted by the disease process.

The case report by Sakakima et al in this issue describes a 53-year-old male with LHCDD diagnosed by the symptom of nephrotic syndrome, hypertension, Ig-G and kappa-chain deposition in glomerulus of kidney, and plasma cell dyscrasia in the bone marrow (3). The clinical course of this case was typical of LHCDD, while there was no beneficial effect on renal function with three VAD therapies, followed by early timing of autologous hematopoietic stem cell transplantation (ASCT), and there may be consideration of renal transplantation in the hemodialysis condition, because he showed disease stability of over one year and no evidence of clinically significant extrarenal deposition.

See also p 970.

The prognosis may be changed by the choice of whether performed early, as first-line therapy, or late, as rescue ASCT treatment. In a myeloma study, the treatment-related mortalities during the first posttransplant year were $9 \%$ and $14 \%$ in the early and late groups (4). Early ASCT after several conventional treatments may be recommended for the deposition-type LHCDD, amyloidosis and other plasma cell disorders. Increasing evidence indicates that high-dose melphalan followed by ASCT improves the clinical outcome of patients with LHCDD, amyloidosis (5) and other plasma cell disorders; this is especially true of patients younger than age 65 . It was recently reported, however, that stem cell transplantation was not so effective in some patients. Thus, to improve the prognosis under such conditions, high-dose melphalan $\left(140-200 \mathrm{mg} / \mathrm{m}^{2}\right)$ in conjunction with autologous/allogeneic hematopoietic stem cell transplantation needs to be done at an early date while the disease still response to the initial therapy.

Kenshi SUZUKI, MD

Department of Hematology, Japanese Red Cross Medical Center, 4-1-22 Hiroo, Shibuya-ku, Tokyo 150-8935

\section{References}

1) Buxbaum J. Mechanisms of disease: monoclonal immunoglobulin deposition. Amyloidosis, light chain deposition disease, and light and 
heavy chain deposition disease. Hematol Oncol Clin North Am 6: 323346, 1992.

2) Gallo GR, Lazowski P, Kumar A, et al. Renal and cardiac manifestations of B-cell dyscrasias with nonamyloidotic monoclonal light chain and light and heavy chain deposition diseases. Adv Nephrol Necker Hosp 28: 355-382, 1998.

3) Sakakima M, Fujigaki Y, Tsuji T, et al. High dose chemotherapy and stem cell support in a patient of light- and heavy-chain deposition disease with abnormal marrow cell surface antigens and no monoclonal protein. Intern Med 44: 970-974, 2005.

4) Fermand JP, Ravaud P, Chevret S, et al. High-dose therapy and autologous peripheral blood stem cell transplantation in multiple myeloma: Up-front or rescue treatment? Results of a multicenter sequential randomized clinical trial. Blood 92: 3131-3136, 1998.

5) Skinner M, Sanchorawala V, Seldin DC, et al. High-dose melphalan and autologous stem-cell transplantation in patients with $\mathrm{AL}$ amyloidosis: An 8-year study. Ann Intern Med 140: 85-93, 2004. 